\title{
Low-Temperature Properties of Quasi-One-Dimensional Molecule-Based Ferromagnets
}

\author{
Takashi Nakanishi, Shoji Yamamoto and Tôru Sakai* \\ Department of Physics, Okayama University, Tsushima, Okayama 700-8530, Japan \\ ${ }^{*}$ Faculty of Science, Himeji Institute of Technology, Ako, Hyogo 678-1297, Japan
}

(12 December 2000)

\begin{abstract}
Quantum and thermal behaviors of low-dimensional mixed-spin systems are investigated with particular emphasis on the design of molecule-based ferromagnets. One can obtain a molecular ferromagnet by assembling molecular bricks so as to construct a low-dimensional system with a magnetic ground state and then coupling the chains or the layers again in a ferromagnetic fashion. Two of thus-constructed quasi-one-dimensional bimetallic compounds are qualitatively viewed within the spin-wave treatment, one of which successfully grows into a bulk magnet, while the other of which ends in a singlet ground state. Then, concentrating on the ferrimagnetic arrangement on a twoleg ladder which is well indicative of general coupled-chain ferrimagnets, we develop the spin-wave theory and fully reveal its low-energy structure. We inquire further into the ferromagnetic aspect of the ferrimagnetic ladder numerically calculating the sublattice magnetization and the magnetic susceptibility. There exists a moderate coupling strength between the chains in order to obtain the most ferromagnetic ferrimagnet.
\end{abstract}

PACS numbers: 75.10.Jm, 75.50.Gg, 75.30.Ds, 75.40.Mg

\section{INTRODUCTION}

Much effort has been devoted to designing molecular systems ordering ferromagnetically and recent progress [i] in the molecular chemistry in this context deserves special mention. One unique approach [2] to molecular ferromagnets consists of assembling molecular bricks so as to construct a low-dimensional system whose ground state possesses a nonzero resultant spin and then coupling the chains or the layers in a ferromagnetic fashion. We are fully convinced that ferromagnetic couplings between nearest-neighbor magnetic centers lead to the highest spin multiplicity, but such an interaction generally requires rather high site symmetries realizing the orthogonality of the magnetic orbitals. Except for a few polynuclear metal complexes [3 6] with the symmetry-imposed orthogonality, most of ferromagnetically coupled molecular systems [7] critically depend on some structural parameters which are hard to handle chemically. An alternative solution [8] to a highly magnetic ground state was obtained from a rather different concept-antiferromagnetically coupled polymetallic systems with irregular spin-state structures. It is the noncompensation of local spins, rather than the ferromagnetic interaction between nearest neighbors, that a magnetic ground state really demands. Ordered bimetallic chain compounds [9] are thus synthesized and since then the magnetic properties of ferrimagnetic chains have extensively been investigated [10 21].

This new strategy for molecular ferromagnets is well described by two contrastive but family compounds 22 24]: $\mathrm{MnCu}($ pba $)\left(\mathrm{H}_{2} \mathrm{O}\right)_{3} \cdot 2 \mathrm{H}_{2} \mathrm{O}$ with pba $=1,3$-propylenebis(oxamato) $=\mathrm{C}_{7} \mathrm{H}_{6} \mathrm{~N}_{2} \mathrm{O}_{6}$ and $\mathrm{MnCu}(\mathrm{pbaOH})\left(\mathrm{H}_{2} \mathrm{O}\right)_{3}$ with pbaOH $=2$-hydroxy-1,3propylenebis(oxamato) $=\mathrm{C}_{7} \mathrm{H}_{6} \mathrm{~N}_{2} \mathrm{O}_{7}$, which are hereafter abbreviated as "pba" and "pbaOH" complexes, re- spectively. Both materials contain one-dimensional alignments of alternating magnetic centers $\mathrm{Mn}$ of $\operatorname{spin} S=\frac{5}{2}$ and $\mathrm{Cu}$ of $\operatorname{spin} s=\frac{1}{2}$ and behave as chain compounds of local spins $S-s$ coupled ferromagnetically. However, due to the interchain interactions, their three-dimensional behaviors are qualitatively different: With decreasing temperature, the divergence of the magnetic susceptibility, which is reminiscent of one-dimensional ferromagnets, is stopped at the onset of three-dimensional ordering for the pba complex, whereas the pbaOH complex still behaves as a ferromagnet below the critical temperature. The former ends up with a singlet ground state in three dimension, while the latter really achieves a molecule-based ferromagnet. Besides the bimetallic chain compounds, certain metal-radical mixed materials [25 27] have also achieved the latter situation.

In such circumstances, Heisenberg alternating-spin chains were fully studied and their ferromagneticantiferromagnetic mixed features [28] were illuminated. However, very little has been calculated beyond one dimension. Namely, theoreticians have indeed assembled molecular bricks so as to construct ferrimagnetic chains but have not yet coupled them in a ferromagnetic fashion. Thus, in this article, we proceed to the latter part of the crystal engineering of molecular ferromagnets-interchain coupling. First, taking the case of the $a b$ planes of the pba and pbaOH complexes, we generally discuss the lowenergy structures of mixed-spin systems beyond one dimension. Second, focusing on the ferromagnetic ordering of the ferrimagnetic chains, we fully investigate two-leg mixed-spin ladders, which are well indicative of general ferrimagnetic coupled-chain systems. Calculating their thermal, as well as quantum, behavior, we finally understand how effective the interchain coupling is in designing molecular ferromagnets. We make full use of the spin-wave theory employing numerical tools as well. 


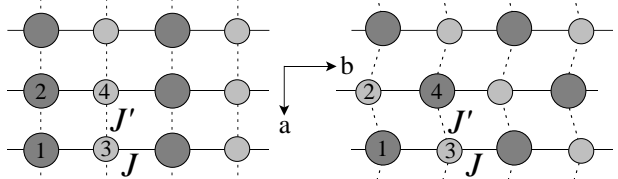

(a)

$\mathrm{Mn} \bigcirc \mathrm{Cu}$

(b)

FIG. 1. Schematic representation of the crystal structures of $\mathrm{MnCu}(\mathrm{pba})\left(\mathrm{H}_{2} \mathrm{O}\right)_{3} \cdot 2 \mathrm{H}_{2} \mathrm{O}(\mathrm{a})$ and $\mathrm{MnCu}(\mathrm{pbaOH})\left(\mathrm{H}_{2} \mathrm{O}\right)_{3}$ (b) in their $a b$ planes.

\section{MIXED-SPIN COUPLED-CHAIN SYSTEMS}

The above-mentioned bimetallic chain compounds, $\mathrm{MnCu}(\mathrm{pba})\left(\mathrm{H}_{2} \mathrm{O}\right)_{3} \cdot 2 \mathrm{H}_{2} \mathrm{O}$ and $\mathrm{MnCu}(\mathrm{pbaOH})\left(\mathrm{H}_{2} \mathrm{O}\right)_{3}$, are schematized in Fig. 1. Due to their characteristic crystal packings 23], the dominant interchain interaction occurs between spins of the same kind in pba, whereas between those of different kinds in pbaOH. Thus these materials may essentially be described by the $l$-leg- $L$-rung mixed-spin coupled-chain Hamiltonians

$$
\mathcal{H}^{(\alpha)}=\sum_{i=1}^{l} \sum_{j=1}^{L}\left(J \boldsymbol{\sigma}_{i, j}^{(\alpha)} \cdot \boldsymbol{\sigma}_{i, j+1}^{(\alpha)}+J^{\prime} \boldsymbol{\sigma}_{i, j}^{(\alpha)} \cdot \boldsymbol{\sigma}_{i+1, j}^{(\alpha)}\right),
$$

where $\mathrm{a}$ and $\mathrm{b}$ are set for the index $\alpha$, corresponding to pba and $\mathrm{pbaOH}$, respectively, and the $j$ th magnetic center on the $i$ th chain, $\boldsymbol{\sigma}_{i, j}^{(\alpha)}$ is explicitly given as $\boldsymbol{\sigma}_{2 m, 2 n-1}^{(\mathrm{a})}=\boldsymbol{\sigma}_{2 m-1,2 n-1}^{(\mathrm{a})}=\boldsymbol{S}, \quad \boldsymbol{\sigma}_{2 m, 2 n}^{(\mathrm{a})}=\boldsymbol{\sigma}_{2 m-1,2 n}^{(\mathrm{a})}=\boldsymbol{s}$,
$\boldsymbol{\sigma}_{2 m-1,2 n-1}^{(\mathrm{b})}=\boldsymbol{\sigma}_{2 m, 2 n}^{(\mathrm{b})}=\boldsymbol{S}, \quad \boldsymbol{\sigma}_{2 m-1,2 n}^{(\mathrm{b})}=\boldsymbol{\sigma}_{2 m, 2 n-1}^{(\mathrm{b})}=\boldsymbol{s}$,

with $S$ and $s$ representing $\mathrm{Mn}(\mathrm{II})$ of spin $S=\frac{5}{2}$ and $\mathrm{Cu}(\mathrm{II})$ of spin $s=\frac{1}{2}$, respectively. We adopt the periodic boundary condition in both leg and rung directions.

In order to qualitatively investigate the low-energy structures, we here make a spin-wave approach to the models. Assuming the Néel configuration and then introducing bosonic spin-deviation operators of four kinds, where the numbering notation is specified in Fig. 1, we obtain the spin-wave Hamiltonians up to quadratic order in the momentum space as

$$
\begin{aligned}
& \mathcal{H}_{\mathrm{SW}}^{(\mathrm{a})}=J \sum_{\boldsymbol{k}}\left[\mathbf{A}_{\boldsymbol{k}}^{\dagger} \mathcal{H}_{\boldsymbol{k}}^{(\mathrm{a})} \mathbf{A}_{\boldsymbol{k}}-4 S s-2 r\left(S^{2}+s^{2}\right)\right], \\
& \mathcal{H}_{\mathrm{SW}}^{(\mathrm{b})}=J \sum_{\boldsymbol{k}}\left[\mathbf{A}_{\boldsymbol{k}}^{\dagger} \mathcal{H}_{\boldsymbol{k}}^{(\mathrm{b})} \mathbf{A}_{\boldsymbol{k}}-4(1+r) S s\right],
\end{aligned}
$$

where

$$
\begin{aligned}
& \mathbf{A}_{\boldsymbol{k}}=\mathrm{T}\left[a_{\boldsymbol{k}}^{(1)} \cdots a_{\boldsymbol{k}}^{(4)} a_{\boldsymbol{k}}^{(1) \dagger} \cdots a_{\boldsymbol{k}}^{(4) \dagger}\right], \\
& \mathcal{H}_{\boldsymbol{k}}^{(\alpha)}=\left[\begin{array}{cc}
\Gamma^{(\alpha)} & \Delta_{\boldsymbol{k}}^{(\alpha)} \\
\Delta_{\boldsymbol{k}}^{(\alpha)} & \Gamma^{(\alpha)}
\end{array}\right],
\end{aligned}
$$

with

$$
\begin{aligned}
& \Gamma^{(\mathrm{a})}=\operatorname{diag}[s+r S, s+r S, S+r s, S+r s], \quad \Delta_{\boldsymbol{k}}^{(\mathrm{a})}=\left[\begin{array}{cccc}
0 & r S \cos \frac{k_{y}}{2} & \sqrt{S s} \cos \frac{k_{x}}{2} & 0 \\
r S \cos \frac{k_{y}}{2} & 0 & 0 & \sqrt{S s} \cos \frac{k_{x}}{2} \\
\sqrt{S s} \cos \frac{k_{x}}{2} & 0 & 0 & r s \cos \frac{k_{y}}{2} \\
0 & \sqrt{S s} \cos \frac{k_{x}}{2} & r s \cos \frac{k_{y}}{2} & 0
\end{array}\right] \text {, } \\
& \Gamma^{(\mathrm{b})}=(1+r) \operatorname{diag}[s, S, S, s], \quad \Delta_{\boldsymbol{k}}^{(\mathrm{b})}=\sqrt{S s}\left[\begin{array}{cccc}
0 & r \cos \frac{k_{y}}{2} & \cos \frac{k_{x}}{2} & 0 \\
r \cos \frac{k_{y}}{2} & 0 & 0 & \cos \frac{k_{x}}{2} \\
\cos \frac{k_{x}}{2} & 0 & 0 & r \cos \frac{k_{y}}{2} \\
0 & \cos \frac{k_{x}}{2} & r \cos \frac{k_{y}}{2} & 0
\end{array}\right] \text {. }
\end{aligned}
$$

Here, we have set twice the lattice constants in the $x$ (leg) and $y$ (rung) directions both equal to unity, whereas $J^{\prime} / J$ equal to $r$. The spin-wave excitations are revealed through the Bogoliubov transformation [29], which can be described in terms of a $8 \times 8$ matrix $\mathcal{U}$ as

$$
\mathbf{A}_{\boldsymbol{k}}=\mathcal{U} \mathbf{B}_{\boldsymbol{k}} ; \mathcal{U} \mathcal{N} \mathcal{U}^{\dagger}=\mathcal{U}^{\dagger} \mathcal{N} \mathcal{U}=\mathcal{N}
$$

where the metric

$$
\mathcal{N}=\operatorname{diag}[1,1,1,1,-1,-1,-1,-1],
$$

has been introduced in order to demand that

$$
\mathbf{B}_{\boldsymbol{k}}=\mathrm{T}\left[b_{\boldsymbol{k}}^{(1)} \cdots b_{\boldsymbol{k}}^{(4)} b_{\boldsymbol{k}}^{(1) \dagger} \cdots b_{\boldsymbol{k}}^{(4) \dagger}\right],
$$

should satisfy the boson commutation relations.
In the case (a) we obtain two spin-wave modes, which are both doubly degenerate, as

$$
\omega_{\sigma}^{(\mathrm{a})}(\boldsymbol{k})=\sqrt{2(A+\sigma \sqrt{B})},
$$

where the index $\sigma$ takes \pm and

$$
\begin{aligned}
& \frac{A}{J^{2}}=\left(S^{2}+s^{2}\right)\left(1+r^{2} \sin ^{2} \frac{k_{y}}{2}\right)+4 S s\left(r-\frac{1}{2} \cos ^{2} \frac{k_{x}}{2}\right), \\
& \frac{B}{J^{4}}=\left(S^{2}-s^{2}\right)^{2}\left(1-r^{2} \sin ^{2} \frac{k_{y}}{2}\right)^{2} \\
& \quad-4 S s \cos ^{2} \frac{k_{x}}{2}\left[(1-r)^{2}(S-s)^{2}-r^{2}(S+s)^{2} \cos ^{2} \frac{k_{y}}{2}\right],
\end{aligned}
$$




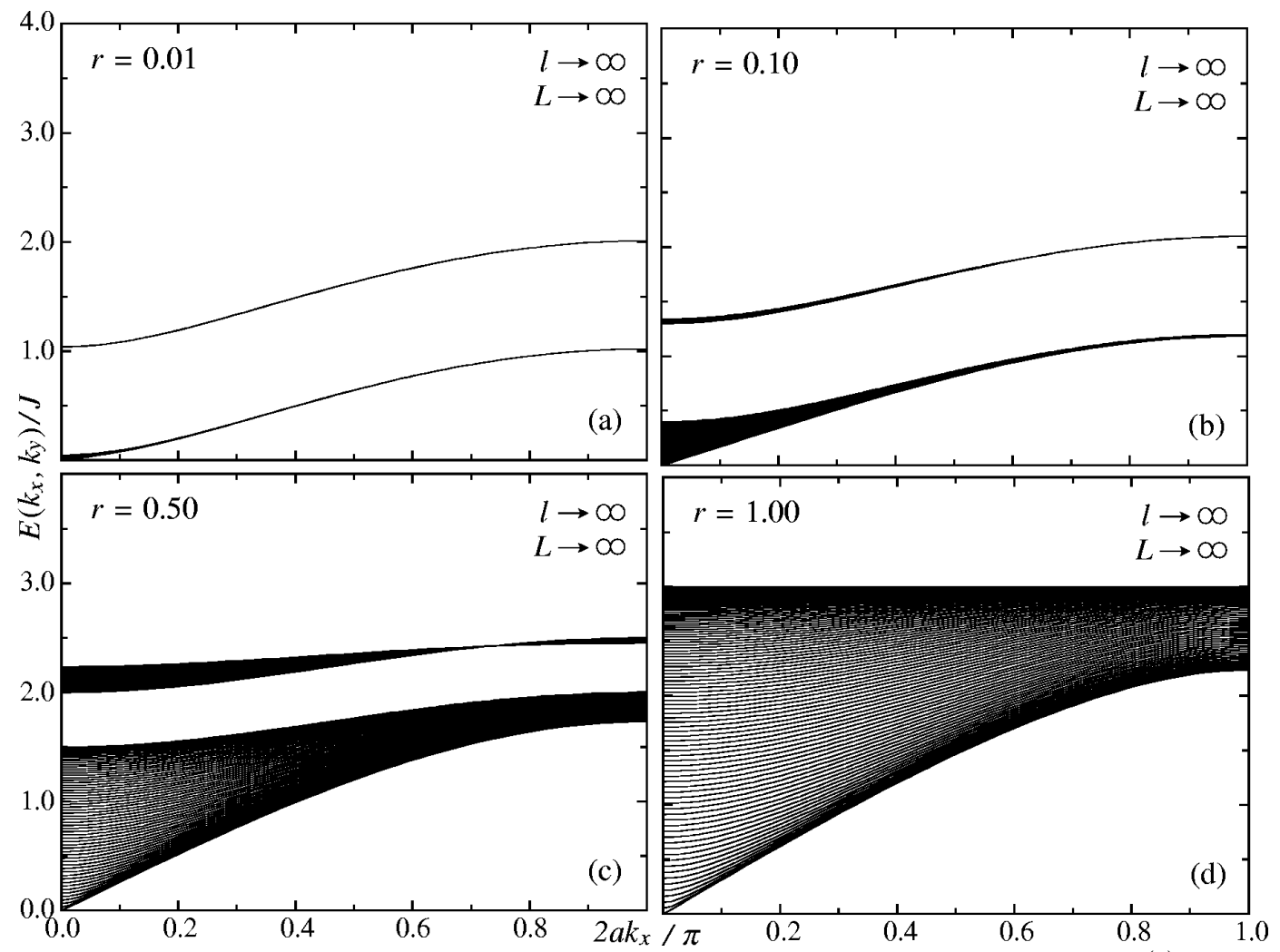

FIG. 2. Dispersion relations of the spin-wave excitations as functions of $k_{x}$ for the Hamiltonian $\mathcal{H}^{(\mathrm{a})}$ with $k_{y}$ fully running in the Brillouin zone and $a$ denoting the lattice constant in the leg direction. $(S, s)$ is set equal to $\left(1, \frac{1}{2}\right)$. $r$ is increased in the order (a) to (d).

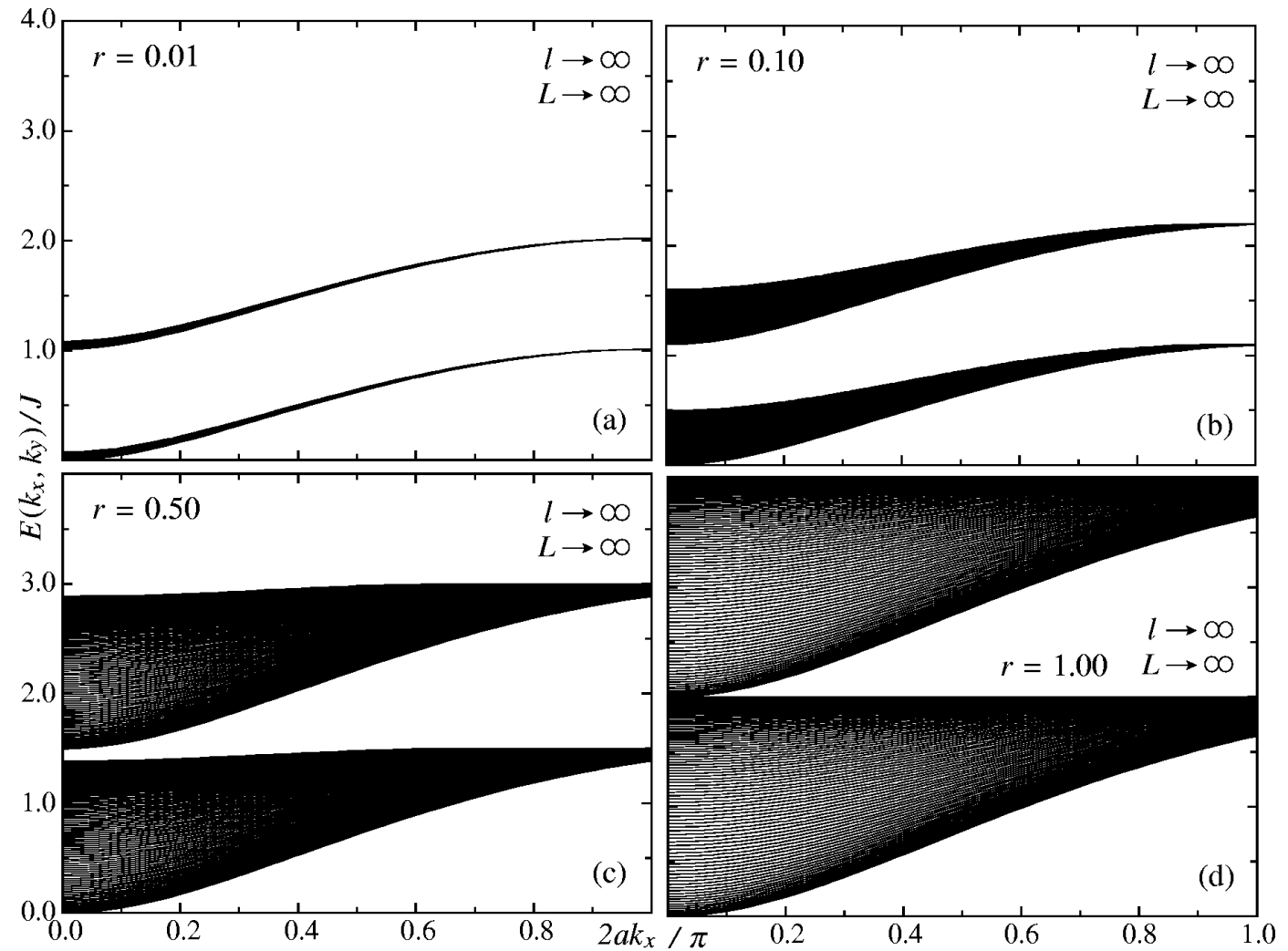

FIG. 3. Dispersion relations of the spin-wave excitations as functions of $k_{x}$ for the Hamiltonian $\mathcal{H}^{(\mathrm{b})}$ with $k_{y}$ fully running in the Brillouin zone and $a$ denoting the lattice constant in the leg direction. $(S, s)$ is set equal to $\left(1, \frac{1}{2}\right) . r$ is increased in the order (a) to (d). 
while in the case (b) the spin waves are generally composed of four modes as

$$
\omega_{\sigma \tau}^{(\mathrm{b})}(\boldsymbol{k})=\sqrt{C-D_{\tau}}+\sigma \sqrt{C},
$$

where the indices $\sigma$ and $\tau$ take \pm and

$$
\begin{aligned}
& \frac{C}{J^{2}}=(1+r)^{2}(S+s)^{2}, \\
& \frac{D_{\tau}}{J^{2}}=4 S s\left(\cos \frac{k_{x}}{2}-\tau r \cos \frac{k_{y}}{2}\right)^{2} .
\end{aligned}
$$

In Figs. 2 and 3 we schematically show the dispersion relations as functions of $k_{x}$ running $k_{y}$ from 0 to $2 \pi$. Since quasi-one-dimensional situations are of our interest, here and in the following we explicitly observe the dispersion in the leg direction. Although the excitation spectra (2.11) and (2.13) look alike in the small- $r$ region, there occurs an essential difference between them with the inclusion of the interchain couplings. In the case (a) the lowest excitation mode exhibits a linear dispersion for small momenta as

$$
\omega_{-}^{(\mathrm{a})}\left(k_{x}, k_{y}=0\right) \sim v_{x}^{(\mathrm{a})} k_{x},
$$

with

$$
v_{x}^{(\mathrm{a})}=J \sqrt{2 r S s \frac{(S-s)^{2}+2(1+r) S s}{(S-s)^{2}+4 r S s}},
$$

whereas in the case (b) it remains quadratic as

$$
\omega_{--}^{(\mathrm{b})}\left(k_{x}, k_{y}=0\right) \sim v_{x}^{(\mathrm{b})} k_{x}^{2},
$$

with

$$
v_{x}^{(\mathrm{b})}=\frac{S s J}{2(S-s)} .
$$

The velocity $v_{x}^{(\mathrm{a})}$ monotonically increases with $r$, while the curvature $v_{x}^{(\text {b) }}$ does not depend on $r$ up to $O\left(S^{1}\right)$. The low-energy physics is much more sensitive to the interchain interaction of the type (a).

Thus, we find that the three-dimensional ground state of the pba complex is singlet regardless of the magnitude of $r$, while the pbaOH complex can be a threedimensional magnet. Systems of the type (a) miss our goal-molecular ferromagnets, but they are interesting in relation to the Haldane-gap problem [30,31]. Coexistence of Haldane-gap excitations and gapless ones induced by the long-range order in $R_{2} \mathrm{BaNiO}_{5}(R=$ magnetic rare earth) [32 36] has been motivating theoretical approaches to the model $\mathcal{H}^{(a)}$ from the $r \rightarrow \infty$ limit 37 40]. Now we restrict ourselves to the model $\mathcal{H}^{(\mathrm{b})}$ and explore further into the low-energy physics essential to ferrimagnetic coupled-chain systems.

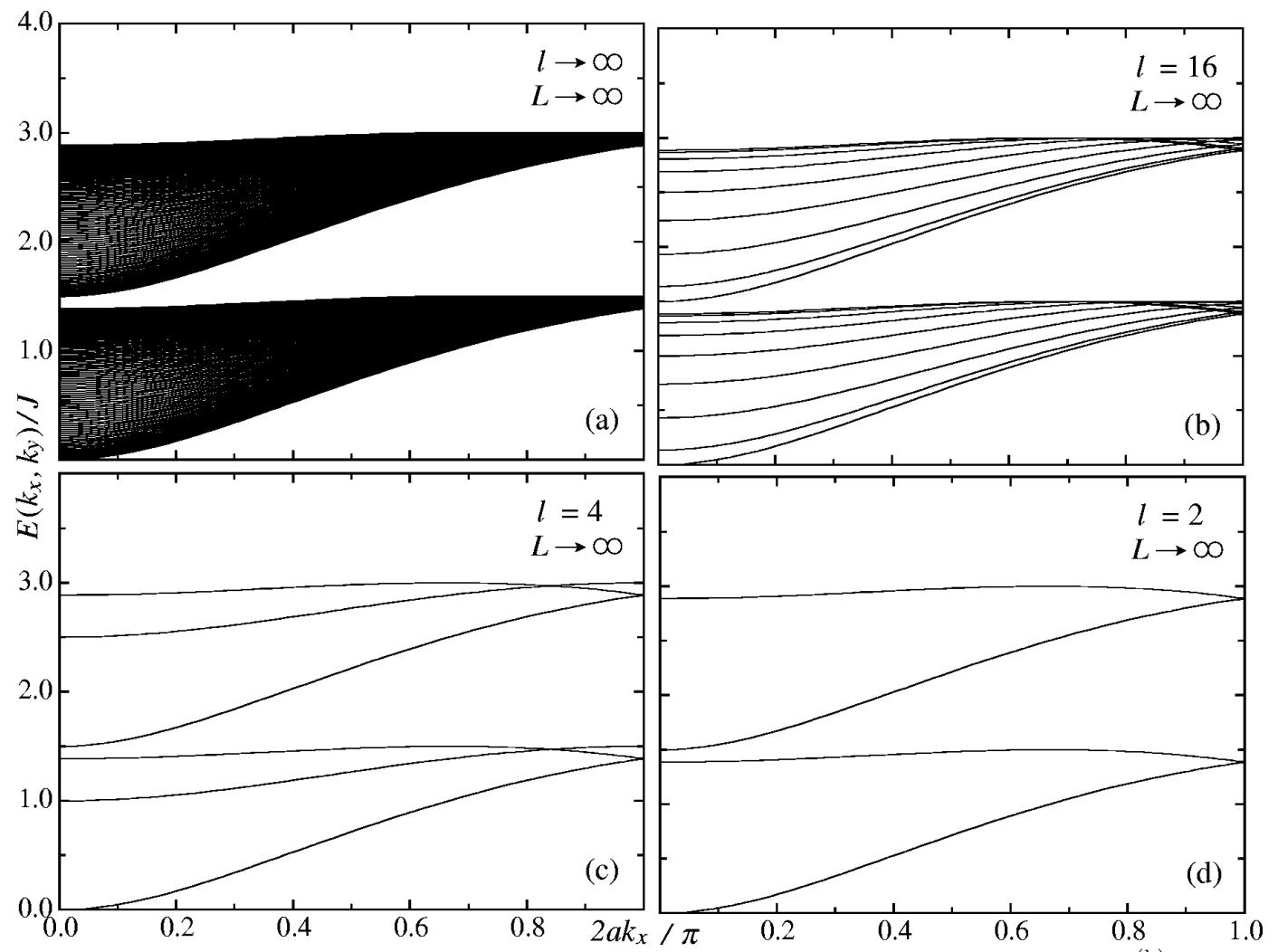

FIG. 4. Dispersion relations of the spin-wave excitations as functions of $k_{x}$ for the Hamiltonian $\mathcal{H}^{(\mathrm{b})}$ with $k_{y}$ taking all the possible values and $a$ denoting the lattice constant in the leg direction. $(S, s)$ is set equal to $\left(1, \frac{1}{2}\right)$, while $r$ equal to 0.5 . The number of legs, $l$, is decreased in the order (a) to (d). 


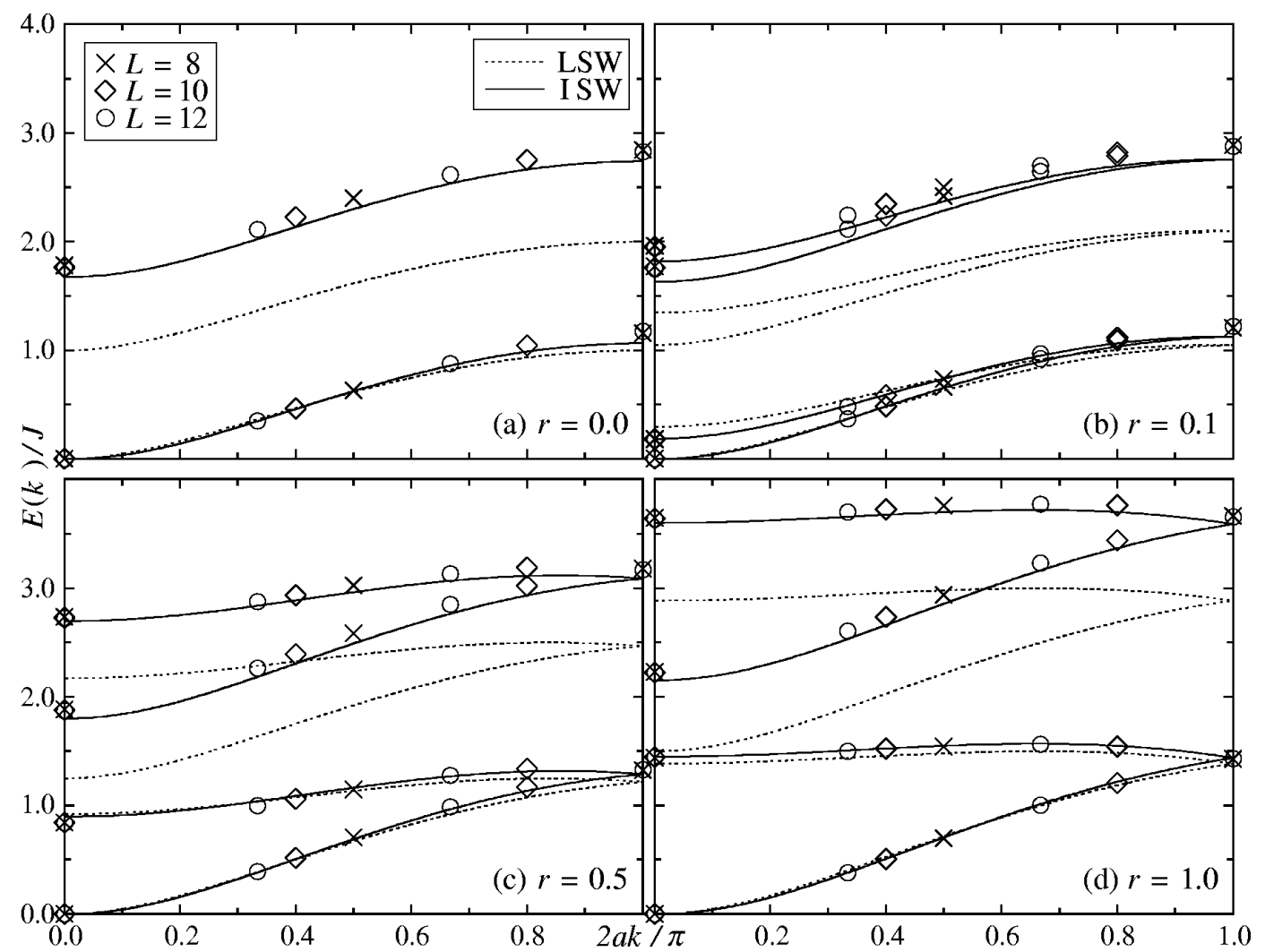

FIG. 5. Dispersion relations of the ferromagnetic and antiferromagnetic elementary excitations, which reduce and enhance the ground-state magnetization and thus lie in the subspaces of magnetization $M=(S-s) L \mp 1$, respectively, for the $(S, s)=\left(1, \frac{1}{2}\right)$ two-leg ferrimagnetic ladders. The noninteracting-spin-wave (LSW) and interacting-spin-wave (ISW) calculations are shown by dotted and solid lines, respectively, whereas symbols represent the exact-diagonalization results. $r$ is increased in the order (a) to (d).

\section{FERRIMAGNETIC SPIN LADDERS}

Considering the present aim of revealing essential effects of the interchain interaction on quasi-onedimensional ferrimagnetic phenomena, further calculations of the two-dimensional lattice are not necessarily efficient. Though numerical tools are indispensable for quantitative understanding, the large degrees of freedom strongly reduce their feasibility. In this context, Fig. 1 gives us a useful piece of information. It is true that the number of states decreases with decreasing $l$, but the essential band structure remains unchanged. Even in the two-leg ladder system, the band width, $\omega_{\sigma+}^{(\mathrm{b})}\left(k_{x}=\right.$ $\left.2 \cos ^{-1} r, k_{y}=0\right)-\omega_{\sigma-}^{(\mathrm{b})}\left(k_{x}=k_{y}=0\right)$, the curvature of the ferromagnetic dispersion, $v_{x}^{(\mathrm{b})}$, and the gap of the lowest antiferromagnetic excitation mode, $\omega_{+-}^{(\mathrm{b})}(0)$, they are all exactly the same as those of the corresponding twoand three-dimensional systems within the up-to- $O\left(S^{1}\right)$ spin-wave theory. The two-leg ferrimagnetic ladder is thus found to be very well indicative of any ferrimagnet in higher dimensions. From the physical point of view, ladder ferrimagnets are highly interesting in themselves, where quantum effects are much more remarkable and therefore novel phenomena are much more expected. There are in fact recent reports [41, 42] on quantum ferri- magnetic spin ladders where quantized plateaux in their ground-state magnetization curves have been revealed.

We proceed to detailed calculation of the low-energy structure of the ferrimagnetic spin ladder. Due to the periodic boundary condition imposed on Eq. (2.1), the two-leg ladder with an intrachain coupling $J$ and an interchain coupling $J^{\prime}$ is described by the Hamiltonian $\mathcal{H}^{(\mathrm{b})}$ with $l=2$ and $J^{\prime}$ being replaced by $J^{\prime} / 2$. For the later convenience, we take $N$ for the number of the elementary units, $L / 2$, and again adopt the notation $r=J^{\prime} / J$. The dispersion relations of the free spin waves are compared with the exact-diagonalization results in Fig. 5 . The lower-bands of ferromagnetic features are very well described by the linear spin-wave theory, whereas the upper-bands of antiferromagnetic features significantly deviate from the free-spin-wave excitations. The relatively poor description of the antiferromagnetic branches by the free spin waves implies that the quantum effect is more relevant in the antiferromagnetic excitations. This is rather convincing when we consider the conventional spin-wave description of Hisenberg ferromagnets and antiferromagnets. The ferromagnetic spin waves correctly describe the low-lying excitations, whereas the antiferromagnetic ones merely give a qualitative view of the low-energy structure leading to the divergence of sublattice magnetizations. Here, the spin-wave theory is much 
more potential and the spin-wave series can in principle reach an accurate description of the antiferromagnetic, as well as ferromagnetic, excitations owing to nondivergent sublattice magnetizations.

In order to refine the up-to- $O\left(S^{1}\right)$ dispersion relations (2.13), let us consider interactions between the spin waves. We may rediagonalize the one-body Hamiltonian (2.3) together with the two-body terms of the order $O\left(S^{0}\right)$ in the naivest attempt to go beyond the linear spin-wave theory. However, such a treatment so misreads the low-energy structure as to bring a gap to the lowest-lying ferromagnetic excitation branch. Therefore, preserving the Bogoliubov transformation, we pick up relevant contributions to the dispersions, as well as to the ground-state energy, from the $O\left(S^{0}\right)$ terms. The Wick theorem allows us to rewrite the spin-wave Hamiltonian of the present two-leg ladder system as

$$
\begin{aligned}
& \mathcal{H}_{\mathrm{SW}}=E_{\text {class }}+E_{0}+E_{1}+\mathcal{H}_{\text {irrel }}+\mathcal{H}_{\text {quart }}+O\left(S^{-1}\right) \\
& \quad+\sum_{\sigma, \tau= \pm} \sum_{k}\left[\omega_{\sigma \tau}(k)+\delta \omega_{\sigma \tau}(k)\right] b_{k}^{(i(\sigma, \tau)) \dagger} b_{k}^{(i(\sigma, \tau))}
\end{aligned}
$$

where the index $i$ of the bosonic operators is a function of $\sigma$ and $\tau$ as $i(\sigma, \tau)=2(\sigma+1) / 2+(\tau+1) / 2+1$, taking 1 to 4 . $\mathcal{H}_{\text {irrel }}$ contains irrelevant terms such as $b_{k}^{(i)} b_{k}^{(j)}$ and $\mathcal{H}_{\text {quart }}$ is the residual two-body interactions in the normal order, both of which are neglected so as to keep the lowest ferromagnetic excitation gapless. $E_{\text {class }}=-4 N J(1+r / 2) S s$ is the Néel-state energy, while $E_{0}$ and $E_{1}$ are the $O\left(S^{1}\right)$ and $O\left(S^{0}\right)$ quantum corrections to it, respectively. $\omega_{\sigma \tau}(k)^{\prime}$ 's are the dispersions for the free spin waves, whereas $\delta \omega_{\sigma \tau}(k)$ 's are the $O\left(S^{0}\right)$ corrections to them. They are all explicitly given in Appendix A. Thus-obtained up-to- $O\left(S^{0}\right)$ dispersion relations potentially gives a precise description of the lowtemperature thermodynamics [28].

The interacting-spin-wave dispersions are also shown in Fig. 5. They excellently describe the antiferromagnetic, as well as ferromagnetic, excitations. We emphasize that the present highly accurate spin-wave description of the low-energy structure is stably obtained for ferrimagnetic chains, ladders, and layers with an arbitrary combination of $S$ and $s$. For $S=s$, where the present system is no more a ferrimagnet, the quantum corrections (A3) and (A5) diverge. This is due to the divergence of the quantum spin reduction

$$
\tau=\frac{1}{N} \sum_{k}\left\langle a_{k}^{(i) \dagger} a_{k}^{(i)}\right\rangle_{\mathrm{g}},
$$

where \langle\rangle$_{\mathrm{g}}$ means the ground-state average. The conventional spin-wave theory 443,44 for low-dimensional Heisenberg antiferromagnets is plagued by the difficulty of the zero-field sublattice magnetizations diverging. Ferrimagnetic systems are generally free from this difficulty and therefore quantum correlations can systematically be calculated. Table II further demonstrates the excellence of the ferrimagnetic spin-wave theory. Unless $r$ is too large to maintain the quasi-one-dimensional aspect, the interchain coupling contributes to the enhancement of the effective dimension of the system. With increasing $r$, the Néel configuration indeed better approximates the ground state. Although the classical configuration considerably deviates from the exact ground state at the decoupled-chain limit $r=0$, the spin-wave series still well describe the ground-state correlations.

\section{FERROMAGNETIC FEATURES OF FERRIMAGNETS}

How should we couple the ferrimagnetic chains in order to grow their ferromagnetic features? We further consider the interchain-coupling effect from this point of view. The ground-state magnetization must be a measure for magnets and it is therefore interesting to observe the quantum spin reduction $\tau$ as a function of $r$. Equation (3.2) is calculated as

$$
\tau=\frac{1}{N} \sum_{k}\left(\psi_{1}^{(2)}(k)^{2}+\psi_{2}^{(2)}(k)^{2}\right),
$$

within the present spin-wave treatment and is plotted in Fig. 6 in comparison with the quantum Monte Carlo estimates. When we consider $\tau$ as a function of constituent spins, Eq. (4.1) monotonically decreases as $S / s$ increases, diverging at the antiferromagnetic limit $S / s=1$ and vanishing for the ferromagnetic limit $S / s \rightarrow \infty$. Though the divergence at $S=s$ is totally due to the HolsteinPrimakoff transformation, this observation of $\tau$ fully justifies the view that $\tau$ should be a measure for the ferromagnetic aspect of ferrimagnets.

The spin waves overestimate quantum fluctuations but they successfully reproduce the minimum of $\tau$ as a function of $r$. When we assemble molecular bricks so as to construct ferrimagnetic chains and then couple them in a ferromagnetic fashion, the Néel configuration grows with increasing $r$ but there is a moderate point $r_{\mathrm{c}}$ to maximize that. For an arbitrary $l$-leg ladder ferrimagnet, $\tau(r \rightarrow \infty)$ is generally larger than $\tau(r=0)$ and thus there should be a minimum of $\tau$ as a function of $r$. The minimum point $r_{\mathrm{c}}$ is smaller than unity as far as $l$ stays finite. If we consider the simple square-lattice plane as is illustrated in Fig. 11, $r_{\mathrm{c}}$ approaches unity for $l \rightarrow \infty$. On the other hand, the recent progress 45] in the molecular chemistry may raise even a possibility of mixed-spin chains based on stable organic radicals forming into a honeycomb lattice, where $r_{\mathrm{c}}$ remains smaller than unity even for $l \rightarrow \infty$. Furthermore the synthesis of a ladder ferrimagnet itself is now indeed in progress [46]. $r_{\mathrm{c}}$ is sensitive to the constituent spins as well as the crystal structure. This point should also be taken into consideration in designing molecular ferromagnets. $r_{\mathrm{c}}$ is an increasing function of the ratio $g \equiv S / s$, suggesting that ferrimagnets should be characterized by $g$ rather than 
$S$ and $s$ themselves. As for the bimetallic chain compounds $\mathrm{MM}^{\prime}(\mathrm{pbaOH})\left(\mathrm{H}_{2} \mathrm{O}\right)_{3} \cdot n \mathrm{H}_{2} \mathrm{O}$, the larger-spin magnetic centers were systematically tuned from $\operatorname{Mn}\left(S=\frac{5}{2}\right)$ to $\mathrm{Ni}(S=1)$, while the smaller-spin ones were mainly fixed to $\mathrm{Cu}\left(s=\frac{1}{2}\right)$ [24]. This is partly due to the problem of crystal engineering but might on the other hand aim at suppressing the quantum spin reduction $\tau$. However, as far as we work along this line, a rather strong interchain coupling is needed to attain the highest transition temperature. If we replace both magnetic centers $\mathrm{M}$ and $\mathrm{M}^{\prime}$ simultaneously, $r_{\mathrm{c}}$ can relatively be reduced at the cost of the amplitude of $\tau$ and therefore the highest transition temperature might be obtained at a smaller feasible interchain coupling.

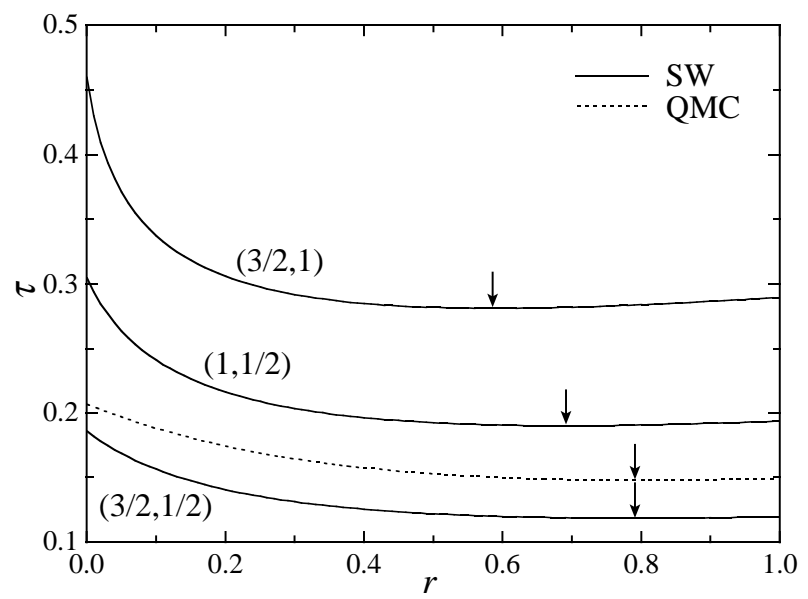

FIG. 6. Spin-wave calculations of the quantum spin reduction $\tau$ as a function of $r$ for the two-leg ferrimagnetic ladders with various values of $(S, s)$. Quantum Monte Carlo estimates are also shown at $(S, s)=\left(1, \frac{1}{2}\right)$ for reference. The minima are indicated by arrows.

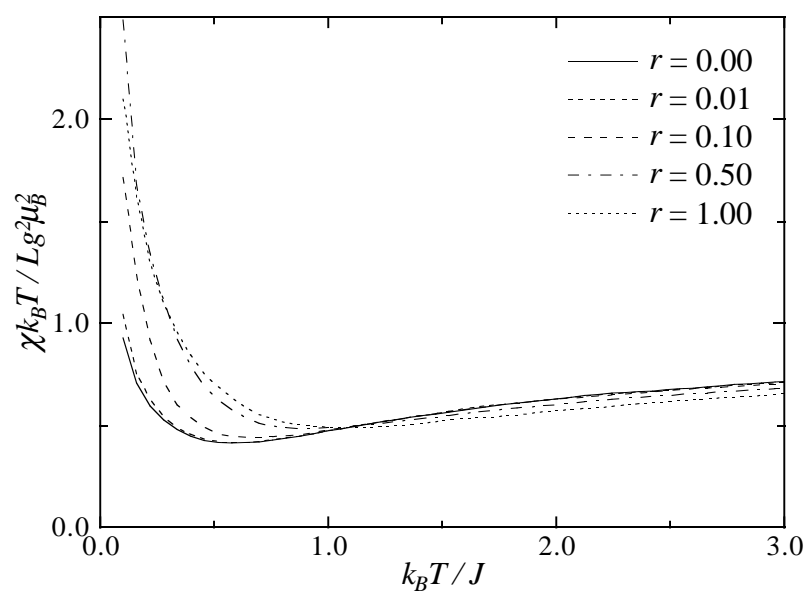

FIG. 7. Quantum Monte Carlo calculations of the temperature dependence of the magnetic susceptibility times temperature for the $(S, s)=\left(1, \frac{1}{2}\right)$ two-leg ferrimagnetic ladders.
In relation to $\tau$, let us observe the ferrimagnetic susceptibility in Fig. 7. The susceptibility-temperature product $\chi T$ is a monotonically decreasing function of $T$ in ferromagnets, while a monotonically increasing function in antiferromagnets. Therefore, the present observation may be regarded as a ferromagnetic-antiferromagnetic mixed nature of ferrimagnets. The low-temperature divergence is really reminiscent of the ferromagnetic susceptibility and therefore signifies how ferromagnetic the ferrimagnet $i s$. Interestingly enough, as $r$ moves away from 0 toward 1 , the diverging behavior is indeed sharpened in the beginning but reaches its limit at a certain value of $r$. The divergence at $r=1$ is clearly duller than that at $r=0.5$. Though the diverging susceptibility is very hard to calculate accurately at low temperatures, the most ferromagnetic ferrimagnet seems to be realized around $r=r_{\mathrm{c}}$.

\section{SUMMARY AND DISCUSSION}

Motivated by the ferrimagnetic chain compounds, we have investigated the magnetic properties of mixed-spin coupled-chain systems featuring a spin-wave analysis. The present spin-wave description is available for general ferrimagnets. It is not only highly successful for quantum correlations but also open to thermal properties complementing numerical investigations. While the small-momentum ferromagnetic excitations should dominate the low-temperature thermodynamics, exactdiagonalization methods are totally unable to describe them. Even with a quantum Monte Carlo technique 477, it is rather hard to obtain the curvature $v$ of their quadratic dispersion relation. The present procedure readily gives $v$ as

$$
\begin{aligned}
& \frac{v}{J}=\frac{S s}{2(S-s)}-\frac{2(S+s)}{N(S-s)} \sum_{k}\left(\psi_{1}^{(2)}(k)^{2}+\psi_{2}^{(2)}(k)^{2}\right) \\
& +\frac{2 \sqrt{S s}}{N(S-s)} \sum_{k}\left(\psi_{1}^{(2)}(k) \psi_{1}^{(4)}(k)+\psi_{2}^{(2)}(k) \psi_{2}^{(4)}(k)\right) \cos \frac{k}{2}
\end{aligned}
$$

It is free from $r$ within the linear spin-wave theory. Its leading $r$ dependence arises from the $O\left(S^{0}\right)$ correction. When $v$ is revealed, we can for instance obtain the leading behavior of the specific heat at low temperatures as

$$
\frac{C}{L k_{\mathrm{B}}}=\frac{3}{16} \zeta\left(\frac{3}{2}\right) \sqrt{\frac{k_{\mathrm{B}} T}{\pi v}}+O\left(\frac{k_{\mathrm{B}} T}{J}\right)
$$

with Riemann's zeta function $\zeta(z)$, provided $r>k_{\mathrm{B}} T / J$. The following terms of the order $O\left(k_{\mathrm{B}} T / J\right)$ can not successfully be obtained within the present spin-wave formulation, but a modified spin-wave theory w8 will bring us them as well as a precise description of the susceptibility. 
The present calculation is not only a theoretical harvest but also encourages further chemical explorations into molecular ferromagnets. While most of thus-farsynthesized ferrimagnets are inorganic-organic hybrid materials, there may be genuine organic ferrimagnets, where the variety and flexibility of the crystal structure, as well as sufficiently small magnetic anisotropy, may enable us to investigate quantum mixed-spin phenomena extensively and essentially. An example approach 49,50 consists of synthesizing an alternating chain of a monoradical and a biradical with a triplet ground state. However, the low-temperature properties of the obtained compounds deviate from the ferrimagnetic behavior suggesting the formation of local singlet clusters. In order to overcome such an inherent difficulty in organic molecule-based materials, another strategy [46] is in progress, where the crystal consists of a triradical containing both spin-1 and spin- $\frac{1}{2}$ units within itself. This material is now believed to possess a ladder-like structure rather than a chain structure predicted in the beginning of the structural analysis. Thus, the first example of a mixed-spin ladder has appeared, though the ferrimag- netic ground state is still not realized there possibly due to next nearest-neighbor interactions.

Interchain interactions inevitably exist in lowdimensional mixed-spin magnets and they seriously affect the formation of the ferrimagnetic ground state. Ladder systems are the simplest but efficient stage to study such phenomena. We expect a close collaboration between theoretical and experimental investigations in designing molecule-based ferromagnets.

\section{ACKNOWLEDGMENTS}

The authors are grateful to Prof. T. Fukui for his useful comments and fruitful discussion. This work was supported by the Japanese Ministry of Education, Science, and Culture through Grant-in-Aid No. 11740206 and by the Sanyo-Broadcasting Foundation for Science and Culture. The numerical computation was done in part using the facility of the Supercomputer Center, Institute for Solid State Physics, University of Tokyo.

\section{APPENDIX A: SPIN-WAVE TREATMENT OF THE FERRIMAGNETIC LADDER}

We express the spin-wave Hamiltonian up to the order $O\left(S^{0}\right)$ as

$$
\mathcal{H}_{\mathrm{SW}}=E_{\text {class }}+E_{0}+E_{1}+\sum_{\sigma, \tau= \pm} \sum_{k}\left[\omega_{\sigma \tau}(k)+\delta \omega_{\sigma \tau}(k)\right] b_{k}^{(i(\sigma, \tau)) \dagger} b_{k}^{(i(\sigma, \tau))}
$$

where

$$
\begin{aligned}
& \frac{E_{0}}{J}=\sum_{k}\left\{\sqrt{\left(1+\frac{r}{2}\right)^{2}(S+s)^{2}-4 S s\left(\cos \frac{k}{2}-\frac{r}{2}\right)^{2}}-\left(1+\frac{r}{2}\right)(S+s)\right. \\
& \left.+\sqrt{\left(1+\frac{r}{2}\right)^{2}(S+s)^{2}-4 S s\left(\cos \frac{k}{2}+\frac{r}{2}\right)^{2}}-\left(1+\frac{r}{2}\right)(S+s)\right\} \\
& \frac{E_{1}}{J}=-\frac{4}{N}\left\{\sum_{k}\left(\psi_{1}^{(2)}(k)^{2}+\psi_{2}^{(2)}(k)^{2}\right)\right\}^{2}-\frac{4}{N}\left\{\sum_{k}\left(\psi_{1}^{(2)}(k) \psi_{1}^{(4)}(k)+\psi_{2}^{(2)}(k) \psi_{2}^{(4)}(k)\right) \cos \frac{k}{2}\right\}^{2} \\
& -\frac{2 r}{N}\left\{\sum_{k}\left(\psi_{1}^{(2)}(k)^{2}+\psi_{2}^{(2)}(k)^{2}\right)\right\}^{2}-\frac{2 r}{N}\left\{\sum_{k}\left(\psi_{1}^{(1)}(k) \psi_{1}^{(2)}(k)+\psi_{2}^{(1)}(k) \psi_{2}^{(2)}(k)\right)\right\}^{2} \\
& +\frac{4}{N}\left(\sqrt{\frac{S}{s}}+\sqrt{\frac{s}{S}}\right) \sum_{k}\left(\psi_{1}^{(2)}(k)^{2}+\psi_{2}^{(2)}(k)^{2}\right) \sum_{k^{\prime}}\left(\psi_{1}^{(2)}\left(k^{\prime}\right) \psi_{1}^{(4)}\left(k^{\prime}\right)+\psi_{2}^{(2)}\left(k^{\prime}\right) \psi_{2}^{(4)}\left(k^{\prime}\right)\right) \cos \frac{k^{\prime}}{2} \\
& +\frac{2 r}{N}\left(\sqrt{\frac{S}{s}}+\sqrt{\frac{s}{S}}\right) \sum_{k}\left(\psi_{1}^{(2)}(k)^{2}+\psi_{2}^{(2)}(k)^{2}\right) \sum_{k^{\prime}}\left(\psi_{1}^{(1)}\left(k^{\prime}\right) \psi_{1}^{(2)}\left(k^{\prime}\right)+\psi_{2}^{(1)}\left(k^{\prime}\right) \psi_{2}^{(2)}\left(k^{\prime}\right)\right), \\
& \frac{\omega_{\sigma \tau}(k)}{J}=\sqrt{\left(1+\frac{r}{2}\right)^{2}(S+s)^{2}-4 S s\left(\cos \frac{k}{2}-\tau \frac{r}{2}\right)^{2}}+\sigma\left(1+\frac{r}{2}\right)(S-s), \\
& \frac{\delta \omega_{\sigma \tau}(k)}{J}=\frac{4}{N}\left\{\left(\sqrt{\frac{S}{s}}+\sqrt{\frac{s}{S}}\right)\left(\psi_{i(\sigma, \tau)}^{(2)}(k) \psi_{i(\sigma, \tau)}^{(4)}(k) \cos \frac{k}{2}+\frac{r}{2} \psi_{i(\sigma, \tau)}^{(1)}(k) \psi_{i(\sigma, \tau)}^{(2)}(k)\right)\right.
\end{aligned}
$$




$$
\begin{aligned}
& \left.-\left(1+\frac{r}{2}\right)\left(\psi_{i(\sigma, \tau)}^{(2)}(k)^{2}+\psi_{i(\sigma, \tau)}^{(4)}(k)^{2}\right)\right\} \sum_{k^{\prime}}\left(\psi_{1}^{(2)}\left(k^{\prime}\right)^{2}+\psi_{2}^{(2)}\left(k^{\prime}\right)^{2}\right) \\
+ & \frac{4}{N}\left\{\sqrt{\frac{S}{s}} \psi_{i(\sigma, \tau)}^{(2)}(k)^{2}+\sqrt{\frac{s}{S}} \psi_{i(\sigma, \tau)}^{(4)}(k)^{2}-2 \psi_{i(\sigma, \tau)}^{(2)}(k) \psi_{i(\sigma, \tau)}^{(4)}(k) \cos \frac{k}{2}\right\} \\
& \times \sum_{k^{\prime}}\left(\psi_{1}^{(2)}\left(k^{\prime}\right) \psi_{1}^{(4)}\left(k^{\prime}\right)+\psi_{2}^{(2)}\left(k^{\prime}\right) \psi_{2}^{(4)}\left(k^{\prime}\right)\right) \cos \frac{k^{\prime}}{2} \\
+ & \frac{2 r}{N}\left\{\sqrt{\frac{S}{S}} \psi_{i(\sigma, \tau)}^{(2)}(k)^{2}+\sqrt{\frac{s}{S}} \psi_{i(\sigma, \tau)}^{(4)}(k)^{2}-2 \psi_{i(\sigma, \tau)}^{(1)}(k) \psi_{i(\sigma, \tau)}^{(2)}(k)\right\} \\
& \times \sum_{k^{\prime}}\left(\psi_{1}^{(1)}\left(k^{\prime}\right) \psi_{1}^{(2)}\left(k^{\prime}\right)+\psi_{2}^{(1)}\left(k^{\prime}\right) \psi_{2}^{(2)}\left(k^{\prime}\right)\right),
\end{aligned}
$$

with

$$
\begin{aligned}
& \psi_{i(\sigma, \tau)}^{(1)}(k)=\frac{\sigma \tau}{R_{i}}\left\{\sqrt{\left(1+\frac{r}{2}\right)^{2}(S+s)^{2}-4 S s\left(\cos \frac{k}{2}-\tau \frac{r}{2}\right)^{2}}-\sigma\left(1+\frac{r}{2}\right)(S+s)\right\}, \\
& \psi_{i(\sigma, \tau)}^{(2)}(k)=\frac{2}{R_{i}}\left(\cos \frac{k}{2}-\tau \frac{r}{2}\right) \sqrt{S s}, \\
& \psi_{i(\sigma, \tau)}^{(3)}(k)=-\frac{2 \tau}{R_{i}}\left(\cos \frac{k}{2}-\tau \frac{r}{2}\right) \sqrt{S s}, \\
& \psi_{i(\sigma, \tau)}^{(4)}(k)=-\frac{\sigma}{R_{i}}\left\{\sqrt{\left(1+\frac{r}{2}\right)^{2}(S+s)^{2}-4 S s\left(\cos \frac{k}{2}-\tau \frac{r}{2}\right)^{2}}-\sigma\left(1+\frac{r}{2}\right)(S+s)\right\}, \\
& R_{i(\sigma, \tau)}^{2}=8 \sigma\left(\cos \frac{k}{2}-\tau \frac{r}{2}\right)^{2} S s-2 \sigma\left\{\sqrt{\left(1+\frac{r}{2}\right)^{2}(S+s)^{2}-4 S s\left(\cos \frac{k}{2}-\tau \frac{r}{2}\right)^{2}}-\sigma\left(1+\frac{r}{2}\right)(S+s)\right\}^{2} .
\end{aligned}
$$

We can obtain the dispersions (A4) by setting $k_{y}$ equal to 0 and replacing $k_{x}$ and $r$ by $k$ and $r / 2$, respectively, in Eq. (2.13). The Bogoliubov transformation is given by Eq. (2.8) with

$$
\mathcal{U}=\left[\begin{array}{cccccccc}
\psi_{1}^{(1)} & \psi_{2}^{(1)} & 0 & 0 & 0 & 0 & -\psi_{3}^{(1)} & -\psi_{4}^{(1)} \\
0 & 0 & \psi_{3}^{(2)} & \psi_{4}^{(2)} & -\psi_{1}^{(2)} & -\psi_{2}^{(2)} & 0 & 0 \\
0 & 0 & \psi_{3}^{(3)} & \psi_{4}^{(3)} & -\psi_{1}^{(3)} & -\psi_{2}^{(3)} & 0 & 0 \\
\psi_{1}^{(4)} & \psi_{2}^{(4)} & 0 & 0 & 0 & 0 & -\psi_{3}^{(4)} & -\psi_{4}^{(4)} \\
0 & 0 & -\psi_{3}^{(1)} & -\psi_{4}^{(1)} & \psi_{1}^{(1)} & \psi_{2}^{(1)} & 0 & 0 \\
-\psi_{1}^{(2)} & -\psi_{2}^{(2)} & 0 & 0 & 0 & 0 & \psi_{3}^{(2)} & \psi_{4}^{(2)} \\
-\psi_{1}^{(3)} & -\psi_{2}^{(3)} & 0 & 0 & 0 & 0 & \psi_{3}^{(3)} & \psi_{4}^{(3)} \\
0 & 0 & -\psi_{3}^{(4)} & -\psi_{4}^{(4)} & \psi_{1}^{(4)} & \psi_{2}^{(4)} & 0 & 0
\end{array}\right]
$$

[1] O. Kahn, Y. Pei and Y. Journaux: in Inorganic Materials, ed. D. W. Bruce and D. O'Hare (Wiley, New York, 1992) p. 95.

[2] J. Miller, J. C. Calabrese, H. Rommelmann, S. R. Chittipeddi, J. H. Zhang, W. M. Reiff and A. J. Epstein: J. Am. Chem. Soc. 109 (1987) 769.

[3] O. Kahn, J. Galy, Y. Journaux, J. Jaud and I. Morgenstern-Badarau: J. Am. Chem. Soc. 104 (1982) 2165.

[4] Y. Journaux, O. Kahn, J. Zarembowitch and J. Galy: J. Am. Chem. Soc. 105 (1983) 7585.
[5] O. Kahn, R. Prins, J. Reedijk and J. S. Thompson: Inorg. Chem. 26 (1987) 3557.

[6] A. Bencini, C. Benelli, A. Dei and D. Gatteschi: Inorg. Chem. 24 (1985) 695.

[7] C. J. Cairns and D. H. Busch: Coord. Chem. Rev. 69 (1986) 1 and references therein.

[8] O. Kahn: Struct. Bonding (Berlin) 68 (1987) 89.

[9] A. Gleizes and M. Verdaguer: J. Am. Chem. Soc. 106 (1984) 3727.

[10] M. Verdaguer, A. Gleizes, J.-P. Renard and J. Seiden: Phys. Rev. B 29 (1984) 5144.

[11] M. Drillon, E. Coronado, R. Georges, J. C. Gianduzzo and J. Curely: Phys. Rev. B 40 (1989) 10992.

[12] S. K. Pati, S. Ramasesha and D. Sen: J. Phys.: Condens. Matter 9 (1997) 8707.

[13] S. Yamamoto, S. Brehmer and H.-J. Mikeska: Phys. Rev. 
B 57 (1998) 13610.

[14] S. Yamamoto: Phys. Rev. B 59 (1999) 1024.

[15] S. Yamamoto, T. Fukui, and T. Sakai: Eur. Phys. J. B. 15 (2000) 211.

[16] S. Yamamoto and T. Sakai: Phys. Rev. B 62 (2000) 3795.

[17] T. Ono, T. Nishimura, M. Katsumura, T. Morita and M. Sugimoto: J. Phys. Soc. Jpn. 66 (1997) 2576.

[18] T. Kuramoto: J. Phys. Soc. Jpn. 67 (1998) 1762.

[19] T. Kuramoto: J. Phys. Soc. Jpn. 68 (1999) 1813.

[20] N. B. Ivanov: Phys. Rev. B 57 (1998) 14024.

[21] C. Wu, B. Chen, X. Dai, Y. Yu and Z.-B. Su: Phys. Rev. B 60 (1999) 1057.

[22] Y. Pei, M. Verdaguer, O. Kahn, J. Sletten and J.-P. Renard, Inorg. Chem. 26 (1987) 138.

[23] O. Kahn, Y. Pei, M. Verdaguer, J.-P. Renard and J. Sletten: J. Am. Chem. Soc. 110 (1988) 782.

[24] P. J. van Koningsbruggen, O. Kahn, K. Nakatani, Y. Pei, J.-P. Renard, M. Drillon and P. Legoll: Inorg. Chem. 29 (1990) 3325.

[25] A. Caneschi, D. Gatteschi, P. Rey and R. Sessoli: Inorg. Chem. 27 (1988) 1756.

[26] A. Caneschi, D. Gatteschi, J.-P. Renard, P. Rey and R. Sessoli: Inorg. Chem. 28 (1989) 1976.

[27] A. Caneschi, D. Gatteschi, J.-P. Renard, P. Rey and R. Sessoli: Inorg. Chem. 28 (1989) 2940.

[28] S. Yamamoto, T. Fukui, K. Maisinger and U. Schollwöck: J. Phys.: Condens. Matter 10 (1998) 11033.

[29] T. Fukui and N. Kawakami: Phys. Rev. B 57 (1998) 398.

[30] F. D. M. Haldane, Phys. Lett. 93A (1983) 464.

[31] F. D. M. Haldane, Phys. Rev. Lett. 50 (1983) 1153.

[32] A. Zheludev, J. M. Tranquada, T. Vogt and D. J. Buttrey: Phys. Rev. B 54 (1996) 6437.

[33] A. Zheludev, J. M. Tranquada, T. Vogt and D. J. Buttrey: Phys. Rev. B 54 (1996) 7210.

[34] T. Yokoo, A. Zheludev, M. Nakamura and J. Akimitsu: Phys. Rev. B 55 (1997) 11516.

[35] T. Yokoo, S. Raymond, A. Zheludev, S. Maslov, E. Ressouche, I. Zaliznyak, R. Erwin, M. Nakamura and J. Akimitsu: Phys. Rev. B 58 (1998) 14424.

[36] S. Raymond, T. Yokoo, A. Zheludev, S. E. Nagler, A. Wildes and J. Akimitsu: Phys. Rev. Lett. 82 (1999) 2382.

[37] S. Maslov and A. Zheludev: Phys. Rev. B 57 (1998) 68.
[38] S. Maslov and A. Zheludev: Phys. Rev. Lett. B 80 (1998) 5786.

[39] A. Koga and N. Kawakami: Phys. Rev. B 61 (2000) 6133.

[40] Y. Takushima, A. Koga and N. Kawakami: Phys. Rev. B 61 (2000) 15189.

[41] A. Langari, M. Abolfath and M. A. Martin-Delgado: Phys. Rev. B 61 (2000) 343.

[42] A. Langari and M. A. Martin-Delgado: Phys. Rev. B 62 (2000) 11725.

[43] P. W. Anderson: Phys. Rev. 86 (1952) 694.

[44] R. Kubo: Phys. Rev. 87 (1952) 568.

[45] Y. Hosokoshi, Y. Nakazawa, K. Inoue, K. Takizawa, H. Nakano, M. Takahashi and T. Goto: Phys. Rev. B 60 (1999) 12924.

[46] Y. Hosokoshi, K. Katoh, K. Inoue and T. Goto: submitted to J. Am. Chem. Soc..

[47] S. Yamamoto: Int. J. Mod. Phys. C 8 (1997) 609.

[48] S. Yamamoto and T. Fukui: Phys. Rev. B 57 (1998) 14008.

[49] D. Shiomi, M. Nishizawa, K. Sato, T. Takui, K. Itoh, H. Sakurai, A. Izuoka and T. Sugawara: J. Phys. Chem. B 101 (1997) 3342.

[50] M. Nishizawa, D. Shiomi, K. Sato, T. Takui, K. Itoh, H. Sawa, R. Kato, H. Sakurai, A. Izuoka and T. Sugawara: J. Phys. Chem. B 104 (2000) 503.

TABLE I. The noninteracting-spin-wave (LSW) and interacting-spin-wave (ISW) estimates of the ground-state energy per rung, $E_{\mathrm{g}} / L J$, for the $(S, s)=\left(1, \frac{1}{2}\right)$ two-leg ferrimagnetic ladders, in comparison with the exact values (Exact) obtained by numerical diagonalization. The classical values (Néel), that is, the energies for the Néel configuration, are also listed for reference.

\begin{tabular}{ccccc}
\hline \hline$r \equiv J^{\prime} / J$ & Néel & LSW & ISW & Exact \\
\hline 0.00 & -1.00 & -1.4365 & -1.4608 & $-1.4542(1)$ \\
0.01 & -1.01 & -1.4371 & -1.4638 & $-1.4566(1)$ \\
0.10 & -1.10 & -1.4539 & -1.4869 & $-1.4807(1)$ \\
0.50 & -1.50 & -1.6270 & -1.6478 & $-1.6459(1)$ \\
1.00 & -2.00 & -1.9295 & -1.9463 & $-1.9433(1)$ \\
\hline \hline
\end{tabular}

